\title{
Las Ferias Agrícolas: Fuente de Diseminación de Nacobbus aberrans $^{1}$
}

Fortunato Rojas, ${ }^{2}$ Javier Franco, ${ }^{3}$ Noel Ortuño ${ }^{3}$

\begin{abstract}
Resumen
Nacobbus aberrans, comúnmente conocido como rosario de la papa, causa severas pérdidas en el rendimiento. Se encuentra en la mayoría de las zonas paperas de Bolivia y su principal medio de diseminación es a través de los tubérculos-semillas infectados. Su presencia en campos del sistema formal de producción de semillas, que sólo cubre el $2 \%$ de la demanda nacional, ocasiona el descenso de categoría o su total descalificación. El resto de tubérculos-semillas que se utilizan para cubrir la demanda nacional proviene del sistema informal, por lo que es importante determinar el potencial de diseminación de $N$. aberrans en tubérculos-semillas procedentes de las ferias regionales que constituyen una de las principales fuentes de comercialización en Cochabamba.
\end{abstract}

Con este objetivo, durante los meses de agosto y setiembre de 1994, que corresponde al período de mayor actividad comercial en las ferias de Arani, Tiraque, Colomi, El Puente, Punata y Quillacollo se recolectó al azar por comerciante y variedad un total de 100 muestras de aproximadamente 10 tubérculos-semillas cada una. La presencia de nematodos en los tubérculos se determinó por maceración y centrifugación de las cáscaras de cinco tubérculos y por el método de bioensayo en bolsa cerrada en los cinco tubérculos restantes. Los resultados obtenidos con ambos métodos indican que

Aceptado para publicación: mayo 1997.

1

2

Egresado de la Facultad de Ciencias Agrícolas y Pecuarias.

Cochabamba, Bolivia.

3 Ph.D. y M.Sc., respectivamente. Nemátologos del Programa de Investigación de la Papa (PROINPA). Convenio: IBTA-CIP-COSUDE, Casilla 4285, Cochabamba, Bolivia. E-mail: proinpa@papa.bo. 
aun cuando se detectó un mayor número de muestras infectadas con el primer método, el segundo es la mejor opción, ya que representa lo que normalmente ocurre con el manejo de semillas y además no requiere de personal ni equipo especializado.

Por otro lado, se determinó que el potencial de diseminación promedio de las muestras en las seis ferias es del $55.7 \%$ con un $28.8 \%$ de tubérculos infectados, sin la influencia la variedad que se comercializa, siempre y cuando los tubérculos-semillas procedan de campos infectados por $N$. aberrans, que en este estudio se estimó en $67.4 \%$.

Palabras claves adicionales: papa, tubérculos infectados, producción de semilla, nematodo, métodos de detección.

\section{Agricultural Fairs: Dissemination Sources for Nacobbus aberrans}

\section{Summary}

Nacobbus aberrans, commonly known as false root-knot nematode, causes severe losses in yield. It is found in most potato growing areas in Bolivia and spreads principally through infected seed tubers. Its presence in fields of the formal seed production system, which supply only $2 \%$ of the national demand, causes a category drop in the crop or total disqualification. The rest of the seed tubers that cover the national demand come from the informal seed system. This shows how important it is to determine the spreading potential of $N$. aberrans in seed tubers from regional fairs, which are one of the main commercial seed supply sources in Cochabamba.

During August and September of 1994, the period of most intense commercial activity, 100 samples of approximately 10 seed tubers each were randomly (as regards variety and supplier) collected from retailers in fairs at Arani Tiraque, Colomi, El Puente, Punata and Quillacollo. Nematode presence in tubers was detected by macerating and centrifuging the skins of five tubers, and by the closed bag bioessay method in the other five. Results obtained by both methods showed that, although a higher number of infected samples were detected

with the first method, the second method proved to be a better option, since it represents the regular seed handling practice and does not require 
specialized personnel or equipment .

On the other hand, it was found that the average spreading or propagation potential in the samples of the six fairs was $55.7 \%$, with $28.8 \%$ of infected tubers in all commercial varieties, when the tubers carne from $N$. aberrans infected fields, which possibility in this study was calculated at $67.4 \%$.

Additional Índex words: $\quad$ potato, infected tubers, seed production, nematodes, detection methods.

El nematodo Nacobbus aberrans comúnmente conocido como el rosario de la papa, probablemente originario de la zona andina de Bolivia y ampliamente distribuido en este país, representa uno de los principales problemas del cultivo de la papa $(2,10)$. Ocasiona pérdidas cuantitativas en el rendimiento $(3,8)$, dependiendo del grado de asociación del hospedante-nematodo, condiciones ambientales y densidad del nematodo, y pérdidas cualitativas en la producción de semilla porque conlleva al descenso de categorías en la certificación de semilla $(5,7)$. Su principal medio de diseminación es el empleo de tubérculos-semillas procedentes de campos infestados hacia otras regiones paperas y a las recientemente incorporadas, a consecuencia del flujo de semilla principalmente a través del sistema no formal (ferias agrícolas) de comercialización. Además, la calidad de la semilla es fundamental para el éxito de cualquier sistema de producción agrícola, especialmente en el cultivo de la papa, porque los tubérculos empleados como semilla son portadores de diversas enfermedades o plagas (6). De esta forma, el propósito del presente estudio fue determinar tanto el potencial de diseminación de $N$. aberrans en tubérculos-semilla procedentes de estas ferias, como el papel de las variedades que se comercializan en relación a sus localidades de origen en la época de mayor actividad comercial de tubérculos-semillas procedentes del sistema no formal de comercialización (1).

\section{Materiales y Métodos}

Durante la última semana de agosto y la primera de setiembre de 1994, que corresponden al período de mayor actividad comercial del sistema informal de semilla de papa, se efectuaron muestreos en las principales ferias agrícolas del Departamento de Cochabamba que se realizan semanalmente en Arani, Tiraque, Colomi, Puente, Púnala y Quillacollo (Figura 1). 
Se recolectó completamente al azar por variedad y por comerciante (agricultor), un total de 100 muestras de aproximadamente 10 tubérculossemilla cada una (Tabla 1). En cada caso, además se realizó una miniencuesta de seis preguntas para obtener información sobre el origen de la semilla y variedad, época de siembra, uso de fertilizantes y pesticidas.

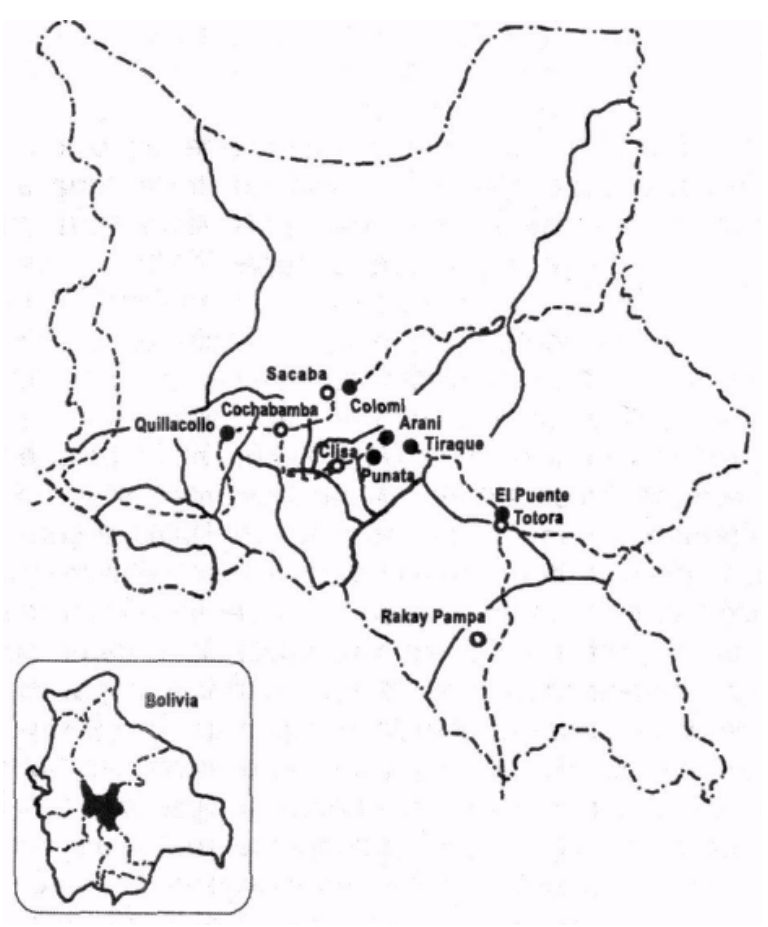

Figura 1. Ubicación de las seis ferias agrícolas estudiadas en el Departamento de Cochabamba, Bolivia. (Modificado de Bustamante, 1992). 
Tabla 1. Proporción de muestras infectadas (con nódulos), porcentaje de tubérculos infectados (potencial de diseminación) y número de nódulos por muestra y tubérculo (severidad) en seis ferias del departamento de Cochabamba, detectados por el método del bioensayo

\begin{tabular}{|c|c|c|c|c|c|c|}
\hline \multirow{2}{*}{ Ferias } & \multirow{2}{*}{$\begin{array}{c}\text { Número } \\
\text { Total de } \\
\text { muestras }\end{array}$} & \multicolumn{2}{|c|}{ Muestras (\%) } & \multirow{2}{*}{$\begin{array}{c}\text { Tubérculos } \\
\text { Infectados } \\
(\%)\end{array}$} & \multicolumn{2}{|c|}{ Número de nódulos } \\
\hline & & Infectada & Libre & & $\begin{array}{c}\text { Muestra } \\
\text { Infectada }\end{array}$ & $\begin{array}{l}\text { Tubérculo } \\
\text { infectado }\end{array}$ \\
\hline Arani & 24 & $16(66.67)$ & $8(33.33)$ & 29.17 & 3.00 & 0.66 \\
\hline Tiraque & 10 & $5(50.00)$ & $5(50.00)$ & 28.00 & ! 1.60 & 0.32 \\
\hline Colomi & 24 & 13(54.17) & $11(45.83)$ & 28.85 & 2.69 & 0.87 \\
\hline Puente & 16 & $8(50.00)$ & $8(50.00)$ & 27.50 & 2.12 & 0.42 \\
\hline Púnala & 10 & $8(80.00)$ & $2(20.00)$ & 25.64 & 2.37 & 0.49 \\
\hline Quillacollo & 12 & $4(33.33)$ & $8(66.67)$ & 33.33 & 2.50 & 0.66 \\
\hline Testigo & 3 & $3(100)$ & -- & 60.00 & 6.00 & 1.20 \\
\hline Media/ferias & & $(55.7)$ & $(44.3)$ & 28.8 & 2.38 & 0.57 \\
\hline
\end{tabular}

La presencia de $N$. aberrans en los tubérculos-semillas se determinó por el método de maceración de cáscaras en licuadora más centrifugación (4) y por el método del bioensayo en bolsa cerrada (9).

Por cada población de tubérculos se separaron cinco tubérculos por método, que constituyeron cinco repeticiones.

\section{Resultados y Discusión}

De acuerdo a los resultados obtenidos entre ambos métodos de detección, el método del maceración de cáscara (licuadora) detectó un mayor porcentaje de muestras infectadas $(79.2 \%)$ en relación al método del bioensayo en bolsa cerrada (56.9\%). Sin embargo, el elevado porcentaje de muestras infectadas detectado por el primer método se debería a que, aun cuando se determinó una mayor cantidad de especímenes presentes en el tubérculo-semilla (activos e inactivos), su infectividad no era óptima por efecto del tipo y tiempo de almacenamiento, el manipuleo y los cambios bruscos de temperatura que sufren éstos en los mercados. 
En cambio, con el método del bioensayo que detectó una menor cantidad de muestras infectadas, éstas representaban la presencia de especímenes activos en el tubérculo-semilla, que se encontraban en condiciones adecuadas para su desarrollo. Además, este último método refleja las condiciones reales del manejo de semilla y no requiere de equipo de laboratorio, ni personal entrenado, para la detección de $N$. aberrans como lo requiere el método de maceración más centrifugación.

Por lo indicado, únicamente los resultados obtenidos mediante el método del bioensayo fueron utilizados para estimar los parámetros de porcentaje de muestras infectadas, potencial de diseminación, y nivel infectivo por muestra y tubérculo.

El porcentaje de muestras infectadas estima la relación entre muestras con $N$. aberrans y el número de muestras colectadas (incidencia en muestras).

El potencial de diseminación expresa el porcentaje de tubérculos infectados por $N$. aberrans en cada una de las muestras colectadas (incidencia en tubérculos).

El nivel infectivo se estimó por el número de nódulos ocasionados por los individuos de $N$. aberrans presentes por tubérculo-semilla o por muestra colectada (severidad).

\section{Comparación entre ferias}

Las ferias de tubérculos-semillas son una importante fuente de diseminación del $N$. aberrans ya que en ellas se comercializa la producción proveniente de diferentes comunidades, cada una con un nivel infectivo muy variable.

Se determinó un mayor porcentaje de infección con $N$. aberrans en muestras provenientes de la feria agrícola de Punata (80.0\%), y más bajo en las muestras provenientes de la feria agrícola de Quillacollo (33.3\%), con un potencial de diseminación de $N$. aberrans de $25.6 \%$ y un nivel infectivo de 0.49 nódulos por tubérculo. Por otro lado, aun cuando el mayor nivel infectivo se observó en las muestras recolectadas en la feria agrícola de Colomi con 0.87 nódulos por tubérculo (Tabla 1), el potencial de diseminación por tubérculos infectados de las seis ferias fue bastante similar (de $25.64 \%$ en Punata a 33.33\% en Quillacollo). Sin embargo, se determinó en promedio de las seis ferias que $55.7 \%$ de las muestras se encontraban infectadas, con un potencial de diseminación de $28.8 \%$. 
De acuerdo a los volúmenes de comercialización de tubérculos -semillas en las ferias de Arani (162.6 t ), Tiraque (102.0 t), Puente (996.7 t) y Quillacollo (250.0 t), la feria agrícola de El Puente resulta con el mayor potencial de diseminación por los grandes volúmenes de tubérculos-semilla que se comercializan.

La baja presencia de $N$. aberrans en muestras recolectadas de la feria de Quillacollo puede atribuirse al origen de las semillas que corresponderían a suelos limpios o con baja infestación, al manipuleo de los tubérculos o a las actividades culturales que se practican en los lugares de origen.

\section{Comparación dentro de ferias según variedad}

La gran mayoría de variedades comercializadas en las ferias presentan niveles infectivos de $N$. aberrans que corresponden al lugar de origen de la semilla, lo que de por sí constituye un alto grado de diseminación del nematodo. Los tubérculos-semillas colectados en la feria agrícola de Arani, que presentaron mayor incidencia de $N$. aberrans fueron los de la variedad Runa Blanca (69.6\%), con un potencial de diseminación neto de $29.2 \%$ y un nivel infectivo de 0.68 nódulos por tubérculo-semilla.

En la feria agrícola de Tiraque se determinó que la mayor incidencia de $N$. aberrans fue en la var. Runa Blanca (66.7\%), con un potencial de diseminación neto de $20 \%$, y un nivel infectivo de 0.3 nódulos por tubérculo, presentando el mayor potencial de diseminación la var. Puka T'ika (40\%) con un nivel infectivo de 0.4 nódulos por tubérculo.

El elevado potencial de diseminación de $N$. aberrans que presentó la variedad Puka T'ika puede atribuirse al origen, que correspondería a suelos de mayor infestación que con la variedad Runa Blanca.

De los tubérculos-semillas colectados en la feria agrícola de Colomi, los que presentaron la mayor incidencia de $N$. aberrans fueron los de las variedades Puka Toralapa, Runa Ch'urku y Pali (100\%), observándose el mayor potencial de diseminación neto en la variedad Waych'a (40\%), con un nivel infectivo de 0.75 nódulos por tubérculo.

Entre los tubérculos-semillas que fueron colectados de la feria agrícola El Puente, los que presentaron una mayor incidencia de $N$, aberrans fueron los de las variedades Puka Toralapa, Yana Waych'a y Holandesa (100\%); el mayor potencial de diseminación lo presentó la variedad Waych'a (35\%), con un nivel infectivo de 0.45 nódulos por tubérculo. 
En la feria agrícola de Punata los tubérculos-semillas que presentaron la mayor incidencia de $N$. aberrans fueron los de la variedad Runa Blanca (88.9\%), con un potencial de diseminación de $25.6 \%$ y un nivel infectivo de 0.48 nódulos por tubérculo-semilla.

Los tubérculos-semillas colectados de la feria agrícola de Quillacollo que presentaron una mayor incidencia de $N$. aberrans correspondían a la variedad Runa Blanca (100\%), con un potencial de diseminación también mayor (42.9\%) y un nivel infectivo de 0.9 nódulos por tubérculo.

Asimismo, los tubérculos-semillas que fueron utilizados como testigo por provenir de campos altamente infestados por $N$. aberrans, presentaron una elevada incidencia (100\%), de igual forma el potencial de diseminación fue alto (60\%), observándose el mayor nivel infectivo en la variedad Waych'a con 1.4 nódulos por tubérculo-semilla (Tabla 2). Estos resultados confirmaron la validez del empleo de la prueba de bioensayo en bolsa cerrada para determinar el potencial o capacidad de diseminación de los tubérculos procedentes de las diversas ferias.

Tabla 2. Proporción de muestras con nódulos, porcentaje de tubérculos infectados, y número de nódulos por muestra y tubérculo según la variedad en muestras testigo

\begin{tabular}{|c|c|c|c|c|c|c|c|}
\hline \multirow[b]{2}{*}{ Variedad } & \multirow{2}{*}{$\begin{array}{c}\text { No. de } \\
\text { muestras }\end{array}$} & \multicolumn{2}{|c|}{ Muestras (\%) } & \multirow{2}{*}{$\begin{array}{l}\text { Tubérculos } \\
\text { infectados } \\
\text { por muestra }\end{array}$} & \multirow{2}{*}{$\begin{array}{c}\text { Tubérculos } \\
\text { Infectados } \\
(\%)\end{array}$} & \multicolumn{2}{|c|}{ No. de nódulos } \\
\hline & & Infectada & libre & & & Muestra & Tubérculos \\
\hline Imilla B. & 1 & $1(100.0)$ & - & $3^{*} / 1^{\star \star}$ & 60.0 & 5.0 & 1.0 \\
\hline Sani I. & 1 & $1(100.0)$ & - & $3^{*} / 1^{\star *}$ & 60.0 & 6.0 & 1.2 \\
\hline Waych'a & 1 & $1(100.0)$ & - & $3^{*} / 1^{\star *}$ & 60.0 & 7.0 & 1.4 \\
\hline
\end{tabular}

* Número de tubérculos infectados por muestra.

** Número de muestras infectadas.

En general, comparando las 6 ferias agrícolas en estudio, de acuerdo a las variedades, se determinó que todas las variedades son potencialmente capaces de diseminar a $N$. aberrans a través de los tubérculos-semillas. La excepción fueron las variedades Gendarme, Yana Imilla y Runa Holandesa (Tabla 3). Pero la ausencia del nematodo en los tubérculos, sobre todo de las dos últimas variedades, se debería a que únicamente se contó con una sola 
muestra de estas variedades. En el caso de la variedad Gendarme el comportamiento era esperado por tratarse de una variedad resistente a $N$. aberrans.

Tabla 3. Porcentaje promedio de tubérculos infectados con N. aberrans en las muestras de las variedades más comunes recolectadas en seis ferias del departamento de Cochabamba.

\begin{tabular}{lcc}
\hline \multicolumn{1}{c}{ Variedades } & No. de muestras & Tubérculos infectados \% \\
\hline Runa Blanca & 41 & 26.7 \\
Waych'a & 24 & 18.1 \\
Puka Tika & 3 & 40.0 \\
Runa Toralapa & 6 & 22.9 \\
Sani Imilla & 10 & 10.0 \\
Runa Churku & 1 & 20.0 \\
Runa Holandesa & 1 & 0.0 \\
Imilla Blanca & 5 & 12.0 \\
Pali & 1 & 20.0 \\
Holandesa & 1 & 20.0 \\
Yana Waych'a & 1 & 20.0 \\
Yana Imilla & 1 & 0.0 \\
Alpha & 1 & 20.0 \\
Gendarme & 1 & 0.0 \\
\hline
\end{tabular}

Por otra parte, se puede notar que los tubérculos-semillas del tipo andigena son los que predominan y parecerían ser los más susceptibles al ataque de N. aberrans, principalmente de las variedades Runa Blanca, Waych'a, Runa, Sani Imilla, etc. Sin embargo, esto se debería esencialmente a que la capacidad o potencial de diseminación de una variedad depende del lugar de origen de la semilla, es decir, el nivel de infestación de los suelos. 


\section{Comparación dentro de las ferias según su procedencia}

La información obtenida a través de las miniencuestas sobre los lugares de origen o procedencia de los tubérculos-semillas que se comercializaban en cada una de las seis ferias, permitieron establecer en cierto grado la probable infestación por $N$. aberrans de estos lugares y que correspondió a $67.4 \%$ de comunidades infestadas y $32.6 \%$ de comunidades libres (Tabla 4).

Tabla 4. Porcentaje de muestras infectadas con Nacobbus aberrans de acuerdo a las comunidades de origen en seis ferias del departamento de Cochabamba.

\begin{tabular}{lccc}
\hline \multicolumn{1}{c}{ Ferias } & $\begin{array}{c}\text { No. de muestras } \\
\text { por origen }\end{array}$ & \multicolumn{2}{c}{ Muestras (\%) } \\
\cline { 2 - 4 } & 12 & $10(83.3)$ & $2(16.7)$ \\
\hline Arani & 6 & $3(50.0)$ & $3(50.0)$ \\
Tiraque & 13 & $9(69.2)$ & $4(30.8)$ \\
Colomi & 7 & $4(57.1)$ & $3(42.9)$ \\
Puente & 8 & $7(87.5)$ & $1(12.5)$ \\
Punata & 7 & $4(57.1)$ & $3(42.9)$ \\
Quillacollo & 53 & $37(67.4)$ & $16(32.6)$ \\
Media de ferias & & & \\
\hline
\end{tabular}

Es así que en la feria de Arani, donde se comercializaban tubérculossemillas procedentes de 12 comunidades, 10 de las muestras fueron positivas, indicando que los 10 lugares de origen se encontraban infestados por $N$. aberrans.

La mayor incidencia de $N$. aberrans se determinó en muestras provenientes de las comunidades de Cerrano Tambo, Wayapacha, Hunt'utuyu, Pedregal, Phuyuphuyu Chico y Molle Molle (100\%), observándose el mayor potencial de diseminación en la comunidad de Cerrano Tambo (66.7\%), con un nivel infectivo de 1 nodulo por tubérculo. No presentaron infección alguna las muestras provenientes de las comunidades de Yanatama y Rodeo.

La ausencia del nematodo $N$. aberrans en las muestras provenientes de la comunidad de Yanatama puede atribuirse en primer lugar a que sólo se contó con una muestra, o también al origen de la semilla que 
corresponderían a suelos limpios o de baja infestación, como resultado de la rotación de cultivos que se practica en la comunidad (papa-trigo-trigodescanso-papa). Del mismo modo se explicaría la ausencia de síntomas radicales (nódulos) en las muestras provenientes de la comunidad de Rodeo.

En la feria de Tiraque, de seis comunidades de origen, tres no mostraron la existencia de $N$. aberrans. De las otras tres, la mayor incidencia de $N$. aberrans se observó en las muestras provenientes de la comunidad de Tiraque (66.7\%), con un potencial de diseminación de 30\% y un nivel infectivo de 0.3 nódulos por tubérculo. El mayor potencial de diseminación se observó en la muestra proveniente de la comunidad de San Isidro (40\%) y un nivel infectivo de 0.4 nódulos por tubérculo. No presentaron infección alguna las muestras provenientes de las comunidades de Sacabambilla y Boquerón K'asa. Por otro lado, no mostraron síntomas radicales las muestras provenientes de la comunidad de Rodeo.

La ausencia del nematodo en las comunidades de Sacabambilla y Boquerón K'asa se atribuiría a las causas mencionadas anteriormente.

De 13 muestras colectadas en la feria de Colomi, las que presentaron mayor incidencia de $N$. aberrans fueron las provenientes de las comunidades de Sta. Bárbara, Chomoco y Kenko (100\%), observándose el mayor potencial de diseminación en muestras provenientes de la comunidad de Troncoli 1 ro (100\%), con un nivel infectivo de 1.5 nódulos por tubérculo. El mayor nivel infectivo se observó en muestras provenientes de Chomoco con 2.6 nódulos por tubérculo-semilla. El elevado nivel infectivo que presenta la comunidad de Chomoco puede atribuirse a suelos altamente infestados como resultado del cultivo intensivo de papa.

Por otro lado, no se observó la presencia del nematodo en tubérculos de las comunidades de Pico, Sora Sora, Cotani y Lluña k'aratuti, que probablemente contarían con suelos libres del nematodo.

De siete comunidades de origen en la feria del Puente y de cuatro que se mostraron positivas, la mayor incidencia de $N$. aberrans se observó en muestras provenientes de la comunidad de Chaupiloma Alto (100\%), con un potencial de diseminación de $20 \%$ y un nivel infectivo de 0.4 nódulos por tubérculo. El mayor potencial de diseminación se observó en la muestra proveniente de la comunidad de Miskha Mayu, con un nivel infectivo de 0.6 nódulos por tubérculo. No se observó la presencia de $N$. aberrans en las muestras provenientes de las comunidades de Laymetoro, Chaupiloma Baja y Mojón. La ausencia del nematodo en muestras provenientes de estas últimas comunidades se atribuiría a que provienen de suelos limpios o con baja infestación debido a la rotación de cultivos que efectúan los agricultores. 
De las ocho muestras recolectadas en la feria de Punata, la proveniente de una comunidad de origen ubicada en el kilómetro 73 se mostró libre y el resto de las muestras presentó una alta incidencia (100\%), observándose el mayor potencial de diseminación en la muestra proveniente de la comunidad de Kilkiluis (60\%), con un nivel infectivo de 1.4 nódulos por tubérculo.

La feria de Quillacollo, con 7 muestras de origen de semilla, presentó la mayor incidencia de $\mathrm{N}$. aberrans en aquellas provenientes de Tiraque y Arani (100\%), observándose el mayor potencial de diseminación en la muestra proveniente de Arani (50\%) con un nivel infectivo de 1.25 nódulos por tubérculo. No se observó la presencia de $N$. aberrans en las muestras provenientes de Colomi y Morochata, porque varias de estas muestras llegaron a podrirse por la mala calidad que presentaban.

De acuerdo a los diversos resultados obtenidos en relación a la semilla que se comercializa, las variedades involucradas o los lugares o comunidades de origen de semilla que se ofertan en las seis ferias muestreadas, se establece que las ferias agrícolas son importantes fuentes de diseminación del nematodo $N$. aberrans, sin influenciar la variedad de tubérculo-semilla que se oferte. Sin embargo, el potencial de diseminación depende esencialmente de los lugares de origen (procedencia) de los tubérculos-semillas, que en el presente estudio se estimó que correspondía a un $67.4 \%$ de comunidades infestadas.

Este trabajo fue ejecutado dentro del marco del convenio del Programa de Investigación de la Papa, PROINPA, establecido entre la Secretaría Nacional de Agricultura y Ganadería de Bolivia, la Cooperación Suiza al Desarrollo (COSUDE) y el Centro Internacional de la Papa (CIP). 


\section{Referencias Bibliográficas}

1. Bustamante, J. 1992. Canales de Comercialización, Intermediarios y Características de la demanda de semilla en Bolivia. En: Informe final de PRACIPA-Comercialización. Lima, Perú, p. 25-51.

2. Caero, G. 1984. Distribución de nematodos de importancia económica en zonas productoras de papa en Bolivia. Tesis Ing. Agr. Cochabamba, Bolivia, UMSS., Facultad de Ciencias Agrícolas y Pecuarias. Cochabamba, Bolivia. 139 p.

3. Canto, M. 1986. Los nematodos y la producción de papa. En: V Curso Internacional sobre el cultivo de papa con énfasis en la producción de semilla. Lima, Perú. p. 193-212.

4. Costilla, M. 1985. El falso nematodo del nodulo Nacobbus aberrans Thorne, 1935; Thorne y Allen, 1944 y su relación con el cultivo de la papa en el Noroeste Argentino. En: Investigaciones Nematológicas en Programas Latinoamericanos de Papa. (Ed. J. Franco y H. Rincón) Centro Internacional de la Papa (CIP): p.3-16.

5. Franco, J. 1994. Problemas de nematodos en la producción de papa en climas templados en la región andina. Nematropica 24:179-195.

6. Gandarillas, A; Revollo, G. 1989. Tecnología para la producción y certificación de semilla de papa en Bolivia CNS. Cochabamba, Bolivia 16 p.

7. Ortuño, N.; Franco, J. 1995. Los nematodos en la producción de semilla de papa en Bolivia. En: Memorias del Curso de Producción de Semilla de Papa IBTA-PROINPA. Cochabamba, Bolivia. 9 p.

8. Otazu, V.; Hoopes, R.; Caero, G.; Huayta, Y. 1985. El rosario de la papa causado por N. aberrans (Thorne, 1933) Thorne y Allen 1944, su efecto en el rendimiento y algunos aspectos que inciden en su propagación y prevalencia en Bolivia. Fitopatología. (Lima-Perú) 20 (2):65-70. 
9. Programa de Investigación de la Papa (PROINPA). 1993. En: Informe Anual 92-93, Cochabamba, Bolivia: p. 216-224

10. Programa de Investigación de la Papa (PROINPA). 1994. En: Informe Anual 93-94, Cochabamba, Bolivia : N2-N3. 